\title{
General Framework for Consistent Sampling in Hilbert Spaces
}

\author{
Yonina C. Eldar \\ Department of Electrical Engineering, \\ Technion-Israel Institute of Technology, Haifa, Israel. \\ yonina@ee.technion.ac.il \\ Tobias Werther* \\ Faculty of Mathematics, \\ University of Vienna, Austria. \\ tobias.werther@univie.ac.at \\ Received (Day Month Year) \\ Revised (Day Month Year) \\ Communicated by (xxxxxxxxxx)
}

\begin{abstract}
We introduce a general framework for consistent linear reconstruction in infinitedimensional Hilbert spaces. We study stable reconstructions in terms of Riesz bases and frames, and generalize the notion of oblique dual frames to infinite dimensional frames. As we show, the linear reconstruction scheme coincides with the so-called oblique projection, which turns into an ordinary orthogonal projection when adapting the inner product. The inner product of interest is, in general, not unique. We characterize the inner products and corresponding positive operators for which the new geometrical interpretation applies.
\end{abstract}

Keywords: Oblique projection; Sampling; Riesz basis; Frames

AMS Subject Classification: 94A20, 46C07

\section{Introduction}

Sampling is the process of representing a signal $f$ by a sequence of numbers, which can be interpreted as measurements of $f$. The classical approach is to choose the measurements as samples of $f$. A more recent approach $1,5,6,7,9,11,12,13,14,15$ is to consider measurements that can be expressed as inner products of $f$ with a set of sampling vectors that span a subspace $\mathcal{S}$, which is referred to as the sampling space. The problem then is to reconstruct $f$ from these measurements, using a set of reconstruction vectors that span a subspace $\mathcal{W}$, which is referred to as the reconstruction space. If $f$ does not lie in $\mathcal{W}$, then it cannot be perfectly reconstructed

${ }^{*}$ The work of the second author was supported by the EU-project NetAGES, IST-1999-29034. 
using only reconstruction vectors that $\operatorname{span} \mathcal{W}$. Therefore, if we allow for signals out of $\mathcal{W}$, we must relax the requirement for perfect reconstruction.

Recently ${ }^{5}$, a general framework for sampling with arbitrary sampling and reconstruction spaces has been developed. This framework is based on a consistency requirement, introduced in the context of sampling in ${ }^{13}$. Specifically, the reconstructed signal has the property that although it is in general not equal to $f$, it nonetheless yields the same measurements. Based on this notion, in ${ }^{5}$ sampling procedures were developed for the case in which $\mathcal{W}$ and $\mathcal{S}$ are finite-dimensional spaces with equal dimension.

In this paper we extend the results of ${ }^{5}$ in two ways. First, we expand the results to a broader framework that does not require $\mathcal{S}$ and $\mathcal{W}$ to be finite-dimensional spaces, but rather can be applied to arbitrary infinite-dimensional subspaces of an arbitrary Hilbert space $\mathcal{H}$ such that $\mathcal{H}=\mathcal{W} \oplus \mathcal{S}^{\perp}$. Throughout the paper we apply the symbol $\oplus$ whenever $\mathcal{W} \cap \mathcal{S}^{\perp}=\{0\}$. We consider both the case of nonredundant sampling and the case of redundant sampling. In both cases we show that consistent reconstruction is always possible, and develop stable reconstruction algorithms, using the concepts of Riesz bases and frames. As we show, the reconstruction schemes are the same as in the finite-dimensional case. We then generalize the notion of the oblique dual frame, introduced in ${ }^{5}$, to infinite-dimensional frames. Specifically, we show that any $w \in \mathcal{W}$ can be expressed as $w=\sum_{i}\left\langle\tilde{s}_{i}, w\right\rangle w_{i}$, where $\left\{w_{i}\right\}$ forms a frame for $\mathcal{W}$, and $\left\{\tilde{s}_{i}\right\}$ forms a frame for $\mathcal{S}$. The frame vectors $\tilde{s}_{i}$ are the oblique dual frames, and have the property that they lead to minimal norm coefficients. The concept of oblique dual frames allows for frame expansions in which the analysis and synthesis frame vectors are not constrained to lie in the same space, as in conventional frame expansions. Complete reconstruction of a subclass of signals by means of frames and dual frames that do not necessarily belong to this subclass is the basic idea of the concept of so-called pseudo-frames ${ }^{8}$. Our motivation of this article, however, is consistency which is not a-priori guaranteed by pseudo-frames.

Second, we develop a new geometric interpretation of the sampling and reconstruction scheme that provides further insight into the problem. Specifically, we show that the linear consistent reconstruction scheme can be expressed as an orthogonal projection when adapting the underlying inner product. The inner product of interest is, in general, not unique. We first characterize the inner products and the corresponding positive operators for which the new geometrical interpretation applies. We then show that by imposing further constraints on the inner product, we can express the reconstruction in a particularly simple form in terms of an orthogonal basis expansion.

The paper is organized as follows. In Section 2, we provide a brief overview of consistent sampling and state necessary and sufficient conditions for the unique existence of the consistent reconstruction. In Section 3, we develop explicit stable reconstruction algorithms, using Riesz bases and frames. In Section 5, we generalize the concept of oblique dual frames to infinite-dimensional spaces. Section 6 considers a geometric interpretation of the sampling and reconstruction. 


\section{Consistent Sampling}

Suppose we are given measurements $c_{i}$ of an unknown signal $f$ belonging to a Hilbert space $\mathcal{H}$, endowed with an inner product $\langle\cdot, \cdot\rangle$. The measurements are assumed to be of the form

$$
c_{i}=\left\langle f, s_{i}\right\rangle
$$

for a set of sampling vector $\left\{s_{i}\right\}$ that span a subspace $\mathcal{S} \subseteq \mathcal{H}$. We want to reconstruct $f$ from the measurements $c_{i}$ using a given set of reconstruction vectors $\left\{w_{i}\right\}$, that span a subspace $\mathcal{W} \subseteq \mathcal{H}$. For designing the reconstruction algorithm we start with the following natural requirements:

(i) uniqueness of the reconstructed signal $\hat{f} \in \mathcal{W}$ with $\left\langle\hat{f}, s_{i}\right\rangle=c_{i}$,

(ii) consistent sampling (interpolation) in the sense that $\left\langle\hat{f}, s_{i}\right\rangle=\left\langle f, s_{i}\right\rangle$.

The first requirement is a requirement on the sampling process. Specifically, we want the sampling vectors $s_{i}$ to be such that if $\left\langle f, s_{i}\right\rangle=\left\langle g, s_{i}\right\rangle$ for all $i$, where $f, g \in \mathcal{W}$, then $f=g$ or, equivalently, for $f, g \in \mathcal{W}$,

$$
\left\langle f-g, s_{i}\right\rangle=0 \Rightarrow f=g .
$$

If $\left\langle f-g, s_{i}\right\rangle=0$ for all $i$, then $f-g \in \mathcal{S}^{\perp}$. Therefore, to satisfy (2.2), we must have that

$$
\mathcal{W} \cap \mathcal{S}^{\perp}=\{0\} .
$$

If the second requirement is also satisfied, so that $\left\langle\hat{f}, s_{i}\right\rangle=\left\langle f, s_{i}\right\rangle$, then for any $f \in \mathcal{W}$, we must have that $\hat{f}=f$. Otherwise we will have two signals $f$ and $\hat{f}$ in $\mathcal{W}$, that have the same measurements, which contradicts the uniqueness requirement.

In our development, we focus our attention on linear reconstruction methods. In this case, the reconstruction $\hat{f}$ is of the form

$$
\hat{f}=\sum_{i} d_{i} w_{i}
$$

for some coefficients $d_{i}$ that are a linear transformation of the measurements $c_{i}$. In other words,

$$
d=H c
$$

for some linear mapping $H$. With $S$ and $W$ denoting the synthesis operators corresponding to $\left\{s_{i}\right\}$ and $\left\{w_{i}\right\}$, respectively, defined by

$$
S: \ell^{2} \rightarrow \mathcal{H}, S c=\sum_{i} c_{i} s_{i},
$$

and

$$
W: \ell^{2} \rightarrow \mathcal{H}, W c=\sum_{i} c_{i} w_{i},
$$

we have that $\hat{f}=W d=W H c=W H S^{*} f$. 
It was shown in ${ }^{5}$, that under the assumption

$$
\mathcal{H}=\mathcal{W} \oplus \mathcal{S}^{\perp},
$$

where $\mathcal{S}$ and $\mathcal{W}$ are closed subspaces of $\mathcal{H}$, a linear reconstruction is consistent if and only if it has the form $\hat{f}=E_{\mathcal{W S}^{\perp}} f$, where $E_{\mathcal{W} \mathcal{S}^{\perp}}$ is the oblique projection onto $\mathcal{W}$ along $\mathcal{S}^{\perp}$, and is the unique operator satisfying

$$
\begin{array}{rlrl}
E_{\mathcal{W S}}{ }^{\perp} & =w, & & (w \in \mathcal{W}), \\
E_{\mathcal{W S}^{\perp}} v & =0, & \left(v \in \mathcal{S}^{\perp}\right) .
\end{array}
$$

Specifically, we have the following theorem.

Theorem 2.1. ${ }^{5}$ Let $\left(\left\langle f, s_{i}\right\rangle\right)$ denote measurements of $f \in \mathcal{H}$ with sampling vectors $\left\{s_{i}\right\}$ that span a subspace $\mathcal{S} \subseteq \mathcal{H}$, and let the reconstruction vectors $\left\{w_{i}\right\}$ span a subspace $\mathcal{W} \subseteq \mathcal{H}$ such that $\mathcal{H}=\mathcal{W} \oplus \mathcal{S}^{\perp}$. Then, $\hat{f}$ is a linear consistent reconstruction of $f$ in $\mathcal{W}$ if and only if $\hat{f}=E_{\mathcal{W} \mathcal{S}^{\perp}} f$.

Theorem 2.1 establishes the existence of consistent reconstruction by means of the oblique projection. Alternatively, we can establish the existence by applying elementary set-theoretical arguments. Specifically, the consistent reconstruction is the unique element of the intersection

$$
\mathcal{W} \cap \mathcal{V}_{f}
$$

where $\mathcal{V}_{f}=f+\mathcal{S}^{\perp}$ is the affine linear space containing all elements in $\mathcal{H}$ that interpolate $\left\langle f, s_{i}\right\rangle$ for all $i$. From the assumption (2.8) it immediately follows that the intersection is not empty and contains only one element, as illustrated in Figure 1.

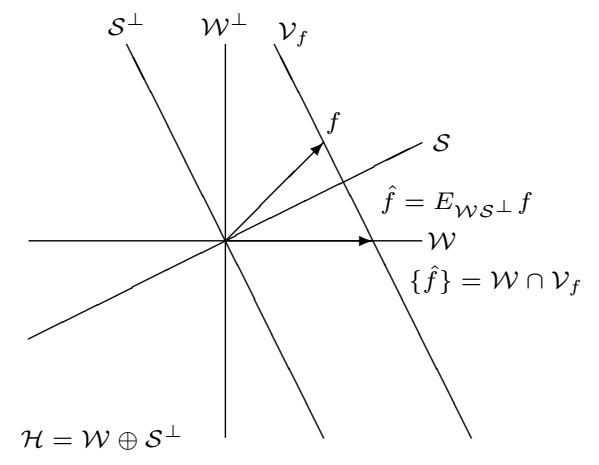

Fig. 1. Illustration of consistent reconstruction.

The viewpoint of the oblique projection will be the key concept of our further development. In order to obtain the reconstruction in practice, we need to develop an explicit reconstruction method, which is the focus of the next section. Explicit reconstruction algorithms for the finite-dimensional case were developed in ${ }^{5}$ both for the case in which $\left\{s_{i}\right\}$ and $\left\{w_{i}\right\}$ are linearly independent vectors, and for the 
case in which they are linearly dependent. For linearly independent vectors, it was shown that consistent reconstruction is obtained with

$$
H=\left(S^{*} W\right)^{-1},
$$

and for linearly dependent sets,

$$
H=\left(S^{*} W\right)^{\dagger},
$$

where $(\cdot)^{\dagger}$ denotes the Moore-Penrose pseudo-inverse.

In the infinite-dimensional case, stability becomes an issue, so that $H$ defined by (2.10) and (2.11) may no longer lead to stable reconstruction algorithms. In the next section we show that using Riesz bases and frames will allow us to overcome instability problems.

\section{Stable Reconstruction}

We now develop stable reconstruction algorithms for the case in which $\mathcal{W}$ and $\mathcal{S}$ are two closed infinite-dimensional subspaces of a Hilbert space $\mathcal{H}$, such that

$$
\mathcal{H}=\mathcal{W} \oplus \mathcal{S}^{\perp} .
$$

To this end, we need to impose conditions on the sampling and reconstruction vectors. We want to emphasize that the splitting of $\mathcal{H}$ in the direct sum (3.1) allows for consistent sampling as stated in Theorem 2.1.

To ensure a stable reconstruction, we assume that the vectors $\left\{s_{i}\right\}$ and $\left\{w_{i}\right\}$ form frames for $\mathcal{S}$ and $\mathcal{W}$, respectively.

Definition 3.1. A family of vectors $\left\{h_{i}\right\}$ in a Hilbert space $\mathcal{H}$ is called a frame for $\mathcal{H}$ if there exist $0<a \leq b<\infty$ such that

$$
a\|h\|^{2} \leq \sum_{i}\left|\left\langle h, h_{i}\right\rangle\right|^{2} \leq b\|h\|^{2},
$$

for all $h \in \mathcal{H}$.

In our derivation, we make use of the following properties of a frame $\left\{h_{i}\right\}^{2}$.

(i) Let $C$ denote the synthesis operator of $\left\{h_{i}\right\}$. The so-called frame-operator $T=$ $C C^{*}$ is invertible on $\mathcal{H}$, and defines the canonical dual frame $\left\{\tilde{h}_{i}\right\}$ through

$$
\tilde{h}_{i}=T^{-1} h_{i} .
$$

(ii) Every element $h \in \mathcal{H}$ can be represented as $h=\sum_{i}\left\langle h, \tilde{h}_{i}\right\rangle h_{i}=\sum_{i}\left\langle h, h_{i}\right\rangle \tilde{h}_{i}$.

(iii) The range of $C^{*}$ is closed in $\ell^{2}$.

In the finite-dimensional case reconstruction is obtained with $H=\left(S^{*} W\right)^{\dagger}$. In general, for arbitrary systems $\left\{s_{i}\right\}$ and $\left\{w_{i}\right\},\left(S^{*} W\right)^{\dagger}$ is not guaranteed to be bounded, which may lead to unstable reconstruction algorithms. However, as we show in the following proposition, if the vectors form a frame, then $\left(S^{*} W\right)^{\dagger}$ is bounded. 
Proposition 3.1. Let $\mathcal{S}$ and $\mathcal{W}$ be closed subspaces of a Hilbert space $\mathcal{H}$ with frames $\left\{s_{i}\right\}$ and $\left\{w_{i}\right\}$ defining the synthesis operators $S$ and $W$, respectively. Then $\mathcal{H}=\mathcal{W} \oplus \mathcal{S}^{\perp}$ if and only if $S^{*} W$ is continuously invertible on $\mathcal{R}\left(W^{*}\right) \rightarrow \mathcal{R}\left(S^{*}\right)$.

Proof. By the frame property (3.), both $\mathcal{R}\left(S^{*}\right)$ and $\mathcal{R}\left(W^{*}\right)$ are closed subspaces of $\ell^{2}$. Therefore, by virtue of the inverse mapping theorem ${ }^{3}$, it suffices to show that $S^{*} W$ is bijective on $\mathcal{R}\left(W^{*}\right) \rightarrow \mathcal{R}\left(S^{*}\right)$.

Let $S^{*} w=0$ with $w=W c$ for some $c \in \mathcal{R}\left(W^{*}\right)$. It follows that $w \in \mathcal{S}^{\perp}$, thus, $w=0$ because of $\mathcal{W} \cap \mathcal{S}^{\perp}=\{0\}$. This implies $c \in \operatorname{Null}(W)=\mathcal{R}\left(W^{*}\right)^{\perp}$. Hence, $c=0$ and $S^{*} W$ is injective.

Let $c \in \mathcal{R}\left(S^{*}\right)$. Then, there exists an element $h=w+s^{\perp} \in \mathcal{H}$ with $S^{*} h=S^{*} w=$ c. Since $\left\{w_{i}\right\}$ constitutes a frame, for $w \in \mathcal{W}$ we can find a sequence $d \in \mathcal{R}\left(W^{*}\right)$ such that $W d=w$. Hence, $S^{*} W d=c$ and $S^{*} W$ is surjective.

Conversely, let $h \in \mathcal{H}$ be decomposed into $h=s_{h}+s_{h}^{\perp}$ according to the orthogonal splitting $\mathcal{H}=\mathcal{S} \oplus \mathcal{S}^{\perp}$. For $\left\{\tilde{s}_{i}\right\}$ be the dual frame of $\left\{s_{i}\right\}$, we have $s_{h}=\sum_{j} c_{j} \tilde{s}_{j}$ with $c=S^{*} s_{h} \in \mathcal{R}\left(S^{*}\right)$. By hypothesis, we can define $w_{h}=W\left(S^{*} W\right)^{-1} c$. It follows that $s_{h}-w_{h} \in \mathcal{S}^{\perp}$ because

$$
S^{*}\left(s_{h}-w_{h}\right)=S^{*} s_{h}-S^{*} W\left(S^{*} W\right)^{-1} c=S^{*} s_{h}-c=0 .
$$

Thus, $h=w_{h}+\left(s_{h}-w_{h}+s_{h}^{\perp}\right)$ is in $\mathcal{W}+\mathcal{S}^{\perp}$.

Let $h \in \mathcal{W} \cap \mathcal{S}^{\perp}$. For $\left\{\tilde{w}_{i}\right\}$ be the dual frame of $\left\{w_{i}\right\}$, we have $h=\sum_{i} c_{i} w_{i}$ with $c_{i}=\left\langle\tilde{w}_{i}, h\right\rangle$. Since $\tilde{w}_{i}=\left(W W^{*}\right)^{-1} w_{i}$, we derive $\left\langle\tilde{w}_{i}, h\right\rangle=\left\langle w_{i},\left(W W^{*}\right)^{-1} h\right\rangle$. Thus, $c \in \mathcal{R}\left(W^{*}\right)$. By hypothesis, $S^{*} W c=S^{*} h=0$ which implies $c=0$, hence, $h=0$. Altogether, we have $\mathcal{H}=\mathcal{W} \oplus \mathcal{S}^{\perp}$.

Due to Proposition 3.1, we can now define the bounded pseudo-inverse operator

$$
\left(S^{*} W\right)^{\dagger}
$$

which coincides with the inverse of $S^{*} W$ on $\mathcal{R}\left(W^{*}\right)$. We claim that

$$
\hat{f}=W\left(S^{*} W\right)^{\dagger} S^{*} f
$$

is the consistent reconstruction of $f$. Indeed, $\hat{f}$ is in $\mathcal{W}$ and from the property of the pseudo-inverse operator that $S^{*} W\left(S^{*} W\right)^{\dagger}$ is the identity on $\mathcal{R}\left(S^{*}\right)$, we have $S^{*} \hat{f}=S^{*} f$. Since all the operators in (3.6) are continuous, the reconstruction is stable.

When considering linearly independent stable systems (Riesz bases), we can replace the pseudo-inverse by the inverse operator just as indicated in (2.10).

Definition 3.2. A family $\left\{h_{i}\right\}$ in a Hilbert space $\mathcal{H}$ is called a Riesz basis of $\mathcal{H}$ if it spans a dense subspace of $\mathcal{H}$ and there exist $0<a \leq b<\infty$ such that

$$
a\|c\|_{\ell^{2}}^{2} \leq\left\|\sum_{i} c_{i} h_{i}\right\|^{2} \leq b\|c\|_{\ell^{2}}^{2}
$$

for all finite sequences $c=\left(c_{i}\right)$. 
For a Riesz basis $\left\{h_{i}\right\}$, the synthesis operator $C: \ell^{2} \rightarrow \mathcal{H}, C\left(c_{i}\right)=\sum_{i} c_{i} h_{i}$, and the analysis operator, which is the adjoint of the synthesis operator, $C^{*}: \mathcal{H} \rightarrow$ $\ell^{2}, C^{*} h=\left(\left\langle h, h_{i}\right\rangle\right)$, are bounded.

For Riesz bases we simply have $\mathcal{R}\left(W^{*}\right)=\mathcal{R}\left(S^{*}\right)=\ell^{2}$ and therefore we obtain the following corollary.

Corollary 3.1. Let $\mathcal{S}$ and $\mathcal{W}$ be closed subspaces of a Hilbert space $\mathcal{H}$ with Riesz bases $\left\{s_{i}\right\}$ and $\left\{w_{i}\right\}$ defining the synthesis operators $S$ and $W$, respectively. Then, $\mathcal{H}=\mathcal{W} \oplus \mathcal{S}^{\perp}$ if and only if $S^{*} W$ is continuously invertible on $\ell^{2}$.

With the assumptions of Corollary 3.1 and $\mathcal{H}=\mathcal{W} \oplus \mathcal{S}^{\perp}$ it can easily be seen that the consistent reconstruction is given by

$$
\hat{f}=W\left(S^{*} W\right)^{-1} c
$$

as in the finite-dimensional case.

Remark 3.1. In contrast to ${ }^{1}$, where the authors treat consistent sampling for the shift invariant case without stating sufficient conditions on stable reconstruction, Proposition 3.1 shows in a more general setting that the reconstruction algorithm is stable if and only if the direct sum condition is satisfied.

\section{Oblique Pseudo Inverse Interpretation}

In ${ }^{5}$, it is shown that the consistent reconstruction $\hat{f}$ can be represented in terms of the oblique pseudo-inverse. We now extend this notion to the infinite-dimensional case. According to ${ }^{4,10}$, we define the oblique pseudo-inverse as follows.

Definition 4.1. Let $T: \mathcal{K} \rightarrow \mathcal{U}$ be a bounded linear transformation between two Hilbert spaces with closed range, and let $\mathcal{K}=\mathcal{G} \oplus \mathcal{N}(T)$ and $\mathcal{U}=\mathcal{R}(T) \oplus \mathcal{Z}$. The oblique pseudo-inverse of $T$ on $\mathcal{G}$ along $\mathcal{Z}$, denoted by $T_{\mathcal{G Z}}^{\#}$, is the unique transformation satisfying

$$
\begin{aligned}
T T_{\mathcal{G} Z}^{\#} & =E_{\mathcal{R}(T) \mathcal{Z}} ; \\
T_{\mathcal{G Z}}^{\#} T & =E_{\mathcal{G N}(T)} ; \\
\mathcal{R}\left(T_{\mathcal{G} \mathcal{Z}}^{\#}\right) & =\mathcal{G} .
\end{aligned}
$$

As can be verified ${ }^{4},(4.1)-(4.3)$ imply that $T_{\mathcal{G} \mathcal{Z}}^{\#}$ inverts $T$ between $\mathcal{G}$ and $\mathcal{R}(T)$, while nulling out any vector in $\mathcal{Z}$. This interpretation is illustrated in Fig. 2, from which it follows that the pseudo-inverse $T^{\dagger}$ is a special case of the oblique pseudoinverse $T_{\mathcal{G} \mathcal{Z}}^{\#}$ for which $\mathcal{G}=\mathcal{N}(T)^{\perp}$ and $\mathcal{Z}=\mathcal{R}(T)^{\perp}$. 


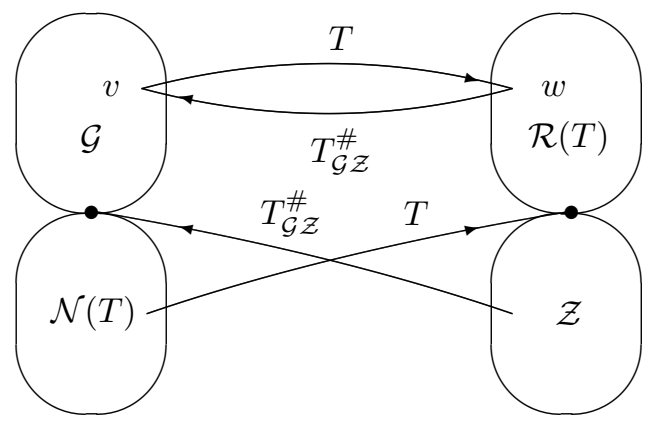

Fig. 2. The action of $T$ and $T_{\mathcal{G} \mathcal{Z}}^{\#}$ on the subspaces $\mathcal{G}, \mathcal{N}(T), \mathcal{R}(T)$ and $\mathcal{Z}$.

In order to show that $\left(S^{*} W\right)^{\dagger} S^{*}$ is the oblique pseudo-inverse of $W$ on $\mathcal{V}=$ $\mathcal{N}(W)^{\perp}$ along $\mathcal{S}^{\perp}$, we have to verify the following properties:

$$
\begin{aligned}
W\left(S^{*} W\right)^{\dagger} S^{*} & =E_{\mathcal{W S}^{\perp}} \\
\left(S^{*} W\right)^{\dagger} S^{*} W & =P_{\mathcal{V}} \\
\left.\mathcal{R}\left(S^{*} W\right)^{\dagger} S^{*}\right) & =\mathcal{V} .
\end{aligned}
$$

This can be verified in the same manner as in ${ }^{5}$. It thus follows that $\hat{f}=W W_{\mathcal{V}_{\mathcal{S}}^{\perp}}^{\#} f$.

\section{Oblique Dual Frame}

The consistent reconstruction framework leads to new frame expansions in which the analysis and synthesis vectors are not constrained to lie in the same space. Specifically, for $\mathcal{S}$ and $\mathcal{W}$ finite-dimensional, it was shown in ${ }^{5}$ that if $\mathcal{H}=\mathcal{W} \oplus \mathcal{S}^{\perp}$, then any $f \in \mathcal{W}$ can be expressed as

$$
f=\sum_{i}\left\langle f, \tilde{s}_{i}\right\rangle w_{i}
$$

where $\tilde{s}_{i}$ are the oblique dual frames of $w_{i}$ on $\mathcal{S}$, and correspond to the synthesis operator $\left(W_{\mathcal{V S}^{\perp}}^{\#}\right)^{*}=S\left(W^{*} S\right)^{\dagger}$. We now show that this definition holds true also in the infinite-dimensional case.

Definition 5.1. Assume that $\mathcal{W}$ and $\mathcal{S}$ are closed subspaces of a Hilbert space $\mathcal{H}$ such that $\mathcal{H}=\mathcal{W} \oplus \mathcal{S}^{\perp}$. Let $\left\{w_{i}\right\}$ and $\left\{s_{i}\right\}$ be frames of $\mathcal{W}$ and $\mathcal{S}$, respectively. The oblique dual frame of $\left\{w_{i}\right\}$ on $\mathcal{S}$ is the frame $\tilde{s}_{i}$ in $\mathcal{S}$ defined by

$$
\tilde{s}_{i}=\left(W_{\mathcal{V} \mathcal{S}^{\perp}}^{\#}\right)^{*} e_{i},
$$

where $\left\{e_{i}\right\}$ denotes the standard basis of $\ell^{2}$ and $\mathcal{V}$ is as in (4.6).

To show that oblique dual frame is well-defined, note that by assumption, $W_{\mathcal{V} \mathcal{S}^{\perp}}^{\#}: l^{2} \rightarrow \mathcal{H}$ is given by $\left(S^{*} W\right)^{\dagger} S^{*}$. Since $\mathcal{R}\left(\left(W_{\mathcal{V} \mathcal{S}^{\perp}}^{\#}\right)^{*}\right)=\mathcal{N}\left(W_{\mathcal{V} \mathcal{S}^{\perp}}^{\#}\right)^{\perp}=\mathcal{S}$, we have $\tilde{s}_{i} \in \mathcal{S}$. Now, $\sum_{i}\left|\left\langle s, \tilde{s}_{i}\right\rangle\right|^{2}=\sum_{i}\left|\left\langle\left(S^{*} W\right)^{\dagger} S^{*} s, e_{i}\right\rangle\right|^{2}=\left\|\left(S^{*} W\right)^{\dagger} S^{*} s\right\|_{\ell^{2}}^{2}$ for 
any $s \in \mathcal{S}$. Since $\left\{s_{i}\right\}$ is a frame and $S^{*} W$ is continuously invertible on $\mathcal{R}\left(S^{*}\right)$ by virtue of Proposition 3.1, there exist positive constants $a$ and $b$ such that

$$
a\|s\|^{2} \leq \sum_{i}\left|\left\langle s, \tilde{s}_{i}\right\rangle\right|^{2} \leq b\|s\|^{2} \quad(s \in \mathcal{S}) .
$$

Hence, $\left\{\tilde{s}_{i}\right\}$ is a frame for $\mathcal{S}$.

The following result reveals the minimal norm property of the canonical dual frame which justifies the choice of terminology.

Proposition 5.1. Assume that $\mathcal{W}$ and $\mathcal{S}$ are closed subspaces of a Hilbert space $\mathcal{H}$ such that $\mathcal{H}=\mathcal{W} \oplus \mathcal{S}^{\perp}$. Let $\left\{w_{i}\right\}$ and $\left\{s_{i}\right\}$ be frames of $\mathcal{W}$ and $\mathcal{S}$, respectively and $\left\{\tilde{s}_{i}\right\}$ the oblique dual frame of $\left\{w_{i}\right\}$ on $\mathcal{S}$. Then, for all possible coefficient sequences $d=\left(d_{i}\right)$ that satisfy

$$
f=\sum_{i} d_{i} w_{i}, \quad(f \in \mathcal{W}),
$$

the coefficient sequence $\tilde{d}=\left\{\left\langle f, \tilde{s}_{i}\right\rangle\right\}$ has minimal $\ell^{2}$-norm

Proof. We compute $\sum_{i}\left\langle f, \tilde{s}_{i}\right\rangle w_{k}=\sum_{i}\left\langle W_{\mathcal{V} \mathcal{S}^{\perp}}^{\#} f, e_{i}\right\rangle w_{i}=W\left(S^{*} W\right)^{\dagger} W^{*} f=$ $E_{\mathcal{W}} \mathcal{S}^{\perp} f$. Thus, $\tilde{d}$ satisfies (5.4). The proof of the minimal norm property is straight forward and can be found in ${ }^{5}$.

\section{Geometrical Interpretation of Consistent Reconstruction}

In the special case in which $\mathcal{W}=\mathcal{S}$, the reconstructed signal $\hat{f}$ is simply the orthogonal projection of $f$ on $\mathcal{W}$, or in other words, it is the minimal norm element of $\mathcal{V}_{f}$. It is therefore not surprising that if we change the inner product of $\mathcal{H}$ such that the oblique projection turns into an orthogonal projection, then the resulting reconstruction is the minimal norm element in $\mathcal{V}_{f}$ for the changed norm.

Definition 6.1. Let $\langle\cdot, \cdot\rangle_{1}$ and $\langle\cdot, \cdot\rangle_{2}$ be two inner products for a Hilbert space $\mathcal{H}$. They are said to be equivalent if there exist $0<a \leq b$ such that

$$
a\langle h, h\rangle_{1} \leq\langle h, h\rangle_{2} \leq b\langle h, h\rangle_{1}, \quad(h \in \mathcal{H}) .
$$

In what follows $A$ always denotes a bounded linear positive operator acting on $\mathcal{H}$. Such an operator defines an equivalent inner product on $\mathcal{H}$ by

$$
\langle h, k\rangle_{A}=\langle h, A k\rangle, \quad(h, k \in \mathcal{H}) .
$$

Conversely, for every equivalent inner product $\langle\cdot, \cdot\rangle_{2}$ there exists a unique operator $A$ such that

$$
\langle h, k\rangle_{2}=\langle h, A k\rangle, \quad(h, k \in \mathcal{H}) .
$$

The operator $A$ can be defined as $T^{*} T$ where $T$ transfers an orthonormal basis with respect to $\langle\cdot, \cdot\rangle_{1}$ into an orthonormal basis with respect to $\langle\cdot, \cdot\rangle_{2}$. 
Our goal now is to look for inner products which transfer $\mathcal{W}$ and $\mathcal{S}^{\perp}$ into orthogonal spaces. The above observations imply that we can turn our attention to continuous positive operators. Suppose that $w_{k}$ is a frame for $\mathcal{W}$. Then we want to define an inner product on $\mathcal{H}$ so that the orthogonal projection onto $\mathcal{W}$ with respect to this inner product is equal to $E_{\mathcal{W S}^{\perp}}$, i.e.

$$
P_{\mathcal{W}}^{A}=W\left(W^{* A} W\right)^{\dagger} W^{* A}=E_{\mathcal{W} \mathcal{S}^{\perp}}
$$

Now, $W^{* A}$ is the unique operator satisfying

$$
\left\langle x, W^{* A} y\right\rangle_{\ell_{2}}=\langle W x, y\rangle_{A}=\langle W x, A y\rangle,
$$

for any $x \in \ell_{2}, y \in \mathcal{H}$. Using the fact that $\langle W x, A y\rangle=\left\langle x, W^{*} A y\right\rangle_{\ell_{2}}$, from (6.5) we have

$$
W^{* A}=W^{*} A .
$$

Since any $w \in \mathcal{W}$ can be written as $w=W a$ for some $a \in \ell_{2}$,

$$
P_{\mathcal{W}}^{A} w=W\left(W^{* A} W\right)^{\dagger} W^{* A} W a=W P_{\mathcal{N}(W)^{\perp}} a=W a=w,
$$

so that to satisfy (6.4) we must have that $W^{* A} v=0$ for any $v \in \mathcal{S}^{\perp}$. From (6.6) this implies that $A v$ must lie in $\mathcal{W}^{\perp}$ for any $v \in \mathcal{S}^{\perp}$. We therefore have the following theorem.

Theorem 6.1. Let $\mathcal{W}$ and $\mathcal{S}^{\perp}$ denote subspaces of a Hilbert space $\mathcal{H}$ such that $\mathcal{H}=\mathcal{W} \oplus \mathcal{S}^{\perp}$, and let $A$ be a positive operator. Then the orthogonal projection onto $\mathcal{W}$ with respect to the inner product $\langle\cdot, \cdot\rangle_{A}$ satisfies $P_{\mathcal{W}}^{A}=E_{\mathcal{W} \mathcal{S}^{\perp}}$ if and only if $A: \mathcal{W} \rightarrow \mathcal{S}$, or equivalently, $A: \mathcal{S}^{\perp} \rightarrow \mathcal{W}^{\perp}$.

The action of $A$ is illustrated in Figure 3.

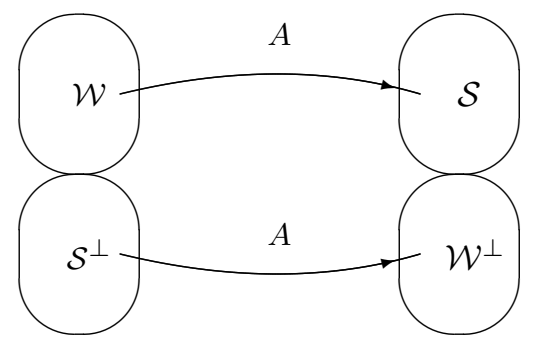

Fig. 3. The action of $A$.

Theorem 6.1 characterizes the class of operators that turn $\mathcal{W}$ and $\mathcal{S}^{\perp}$ into orthogonal spaces. From the theorem it is obvious that the choice of $A$ is not unique. Actually, the class is closed under linear combinations with positive coefficients.

If we choose an inner product for which $E_{\mathcal{W S}^{\perp}}$ becomes an orthogonal projection, then $\hat{f}$ is a minimal norm approximation to $f$ with respect to this inner product. Specifically, for any $w \in \mathcal{W}$,

$$
\|f-\hat{f}\|_{A} \leq\|f-w\|_{A} .
$$


We conclude that the oblique projection performs an orthogonal projection with respect to a whole class of inner products. In other words, the consistent solution is the minimal norm interpolation of the measurements $c_{i}$ for the corresponding sampling vectors $s_{i}$ with respect to any norm defined by a positive operator $A$ of the form illustrated in Figure 3. The interesting observation is that, although we have many different but equivalent norms, the minimal norm interpolation always remains the same.

Assume that we have a Riesz basis $\left\{w_{i}\right\}$ for $\mathcal{W}$ whose synthesis operator will be denoted by $W$. Our next goal is to find an inner product on $\mathcal{H}$ which turns $\left\{w_{i}\right\}$ into an orthonormal basis of $\mathcal{W}$, out of the class of all inner products which make $\mathcal{W}$ orthogonal to $\mathcal{S}^{\perp}$. If $A$ defines such an inner product, then we have

$$
E_{\mathcal{W} \mathcal{S}^{\perp}}=P_{\mathcal{W}}^{A}=W W^{*} A=\sum_{i}\left\langle\cdot, w_{i}\right\rangle_{A} w_{i}
$$

For Riesz bases $\left\{s_{i}\right\},\left\{w_{i}\right\},\left\{s_{i}^{\perp}\right\}$, and $\left\{w_{i}^{\perp}\right\}$ of $\mathcal{S}, \mathcal{W}, \mathcal{S}^{\perp}$, and $\mathcal{W}^{\perp}$, respectively, with corresponding synthesis operator $S, W, S^{\perp}$, and $W^{\perp}$, simple linear algebra arguments induce that the operator

$$
A=S\left(W^{*} S\right)^{-1}\left(S^{*} W\right)^{-1} S^{*}+W^{\perp}\left(S^{\perp *} W^{\perp}\right)^{-1}\left(W^{\perp *} S^{\perp}\right)^{-1} W^{\perp *}
$$

defines an inner product on $\mathcal{H}$ for which $\left\{w_{i}\right\}$ turns into an orthonormal basis and $\mathcal{W}$ is orthogonal to $\mathcal{S}^{\perp}$. This explicitly shows how one can construct such a positive operator.

There are many other operators $A$ satisfying (6.9). However, it is interesting to observe that the action of such operators on $\mathcal{W}$ is unique. Indeed, since for any $w \in \mathcal{W}, w=W W^{*} A w=W W^{*} B w$, we have that $A w=B w$ for all $w \in \mathcal{W}$. Thus any operator $B$ satisfying $E_{\mathcal{W} \mathcal{S}^{\perp}}=W W^{*} B$ must be of the form

$$
B=S\left(W^{*} S\right)^{-1}\left(S^{*} W\right)^{-1} S^{*}+C,
$$

where $C w=0$ for any $w \in \mathcal{W}$. In addition, to satisfy Theorem 6.1, $C$ must map $\mathcal{S}^{\perp}$ to $\mathcal{W}^{\perp}$. We therefore have the following theorem.

Theorem 6.2. Let $\mathcal{W}$ and $\mathcal{S}^{\perp}$ denote subspaces of a Hilbert space $\mathcal{H}$ such that $\mathcal{H}=\mathcal{W} \oplus \mathcal{S}^{\perp}$, let $\left\{w_{i}\right\}$ denote a Riesz basis for $\mathcal{W}$ corresponding to $W$, and let $A$ denote an arbitrary positive operator. Then $E_{\mathcal{W} \mathcal{S}^{\perp}}=P_{\mathcal{W}}^{A}=W W^{*} A$ if and only if $A=S\left(W^{*} S\right)^{-1}\left(S^{*} W\right)^{-1} S^{*}+C$ where $C: \mathcal{W} \rightarrow\{0\}$ and $C: \mathcal{S}^{\perp} \rightarrow \mathcal{W}^{\perp}$.

Note that any operator $A$ satisfying the requirements of Theorem 6.2 has the additional property that $W^{*} A$ is the oblique pseudo-inverse on $\mathcal{V}=\mathcal{N}(W)^{\perp}$ along $\mathcal{S}^{\perp}$. To see this we have to verify that

$$
\begin{aligned}
W W^{*} A & =E_{\mathcal{W S}}, \\
W^{*} A W & =P \mathcal{V}, \\
\mathcal{R}\left(W^{*} A\right) & =\mathcal{V} .
\end{aligned}
$$


Property (6.12) is (6.9). The second property follows from the easily verifiable fact that $W^{*} A W(\mathcal{V})=\mathcal{V}$ and $W^{*} A W\left(\mathcal{V}^{\perp}\right)=\{0\}$. Property (6.14) is a consequence of $\mathcal{R}\left(W^{*} A\right)=\mathcal{R}\left(W^{*}\right)=\mathcal{N}(W)^{\perp}=\mathcal{V}$ which is fulfilled for any positive operator $A$.

Let $\left\{s_{i}\right\}$ be a Riesz basis for $\mathcal{S}$. If we want to linearly reconstruct a signal $f \in \mathcal{H}$ in $\mathcal{W}$ from the samples $\left\langle f, s_{i}\right\rangle$ requiring consistency, then we recall that

$$
\hat{f}=E_{\mathcal{W} \mathcal{S}^{\perp}} f=W\left(S^{*} W\right)^{-1}\left(S^{*} f\right),
$$

which is equivalent to the orthogonal projection

$$
\hat{f}=P_{\mathcal{W}}^{A} f=\sum_{i}\left\langle f, A w_{i}\right\rangle w_{i}
$$

for an operator $A$ satisfying the properties of Theorem 6.2. Here, we see that for reconstruction, $A$ needs only to be applied on $\mathcal{W}$, so that for all practical purposes $A$ is actually unique.

Acknowledgements. The authors would like to thank the anonymous reviewers and H. G. Feichtinger for many constructive comments and ideas for improvement.

\section{References}

1. A. Aldroubi and M. Unser. Sampling procedures in function spaces and asymptotic equivalence with Shannon's sampling theory. Numer. Funct. Anal. Optimiz., 15:1-21, Feb. 1994.

2. O. Christensen. An introduction to frames and Riesz bases. Birkhäuser, 2003.

3. J. B. Conway. A Course in Functional Analysis. Springer-Verlag, 1985.

4. Y. C. Eldar. Quantum Signal Processing. PhD thesis, Massachusetts Institute of Technology, Dec. 2001.

5. Y. C. Eldar. Sampling and reconstruction in arbitrary spaces and oblique dual frame vectors. J. Fourier Analys. Appl., 9(1):77-96, Jan. 2003.

6. Y. C. Eldar. Sampling without input constraints: Consistent reconstruction in arbitrary spaces. In Sampling, Wavelets and Tomography. A. Zayed and J. J. Benedetto, to appear.

7. Y. C. Eldar and A. V. Oppenheim. Nonredundant and redundant sampling with arbitrary sampling and reconstruction spaces. Proceedings of the 2001 Workshop on Sampling Theory and Applications, SampTA'01, pages 229-234, May 2001.

8. S. Li and H. Ogawa. A theory of psuedoframes for subspaces with applications. J. Fourier Analys. Appl. to appear.

9. S. G. Mallat. A theory of multiresolution signal decomposition: The wavelet representation. IEEE Trans. Pattern Anal. Mach. Intell., 11:674-693, 1989.

10. R. D. Milne. An oblique matrix pseudoinverse. SIAM J. Appl. Math., 16(5):931-944, Sep. 1968.

11. M. Unser. Splines: A perfect fit for signal and image processing. IEEE Signal Processing Mag., pages 22-38, Nov. 1999.

12. M. Unser. Sampling - 50 years after Shannon. IEEE Proc., 88:569-587, Apr. 2000.

13. M. Unser and A. Aldroubi. A general sampling theory for nonideal acquisition devices. IEEE Trans. Signal Processing, 42(11):2915-2925, Nov. 1994.

14. P. P. Vaidyanathan. Generalizations of the sampling theorem: Seven decades after Nyquist. IEEE Trans. Circuit Syst. I, 48(9):1094-1109, Sep. 2001.

15. P. P. Vaidyanathan and B. Vrcelj. Biorthogonal partners and applications. IEEE Trans. Signal Processing, 49(5):1013-1027, May 2001. 